\title{
UNIQUE-MAXIMUM COLORING OF PLANE GRAPHS
}

\author{
IGOR FABRICI $^{1}$ \\ Institute of Mathematics \\ P.J. Šafárik University in Košice \\ Slovakia \\ AND \\ FRANK GÖRING \\ Fakultät für Mathematik \\ Technische Universität Chemnitz \\ Germany
}

\begin{abstract}
A unique-maximum $k$-coloring with respect to faces of a plane graph $G$ is a coloring with colors $1, \ldots, k$ so that, for each face $\alpha$ of $G$, the maximum color occurs exactly once on the vertices of $\alpha$. We prove that any plane graph is unique-maximum 3-colorable and has a proper unique-maximum coloring with 6 colors.
\end{abstract}

Keywords: plane graph, weak-parity coloring, unique-maximum coloring. 2010 Mathematics Subject Classification: 05C15.

\section{REFERENCES}

[1] J.A. Bondy and U.S.R. Murty, Graph Theory (Springer, 2008).

[2] D.P. Bunde, K. Milans, D.B. West and H. Wu, Parity and strong parity edge-coloring of graphs, Congr. Numer. 187 (2007) 193-213.

[3] P. Cheilaris, Conflict-Free Coloring, PhD Thesis (City University of New York, 2009).

\footnotetext{
${ }^{1}$ This work was supported by the Slovak Science and Technology Assistance Agency under the contract No. APVV-0023-10, by Grant VEGA 1/0652/12, and by the Agency of the Slovak Ministry of Education for the Structural Funds of the EU under project ITMS:26220120007.
} 
[4] P. Cheilaris, B. Keszegh and D. Pálvölgyi, Unique-maximum and conflict-free colouring for hypergraphs and tree graphs, SIAM J. Discrete Math. 27 (2013) 1775-1787. doi:10.1137/120880471

[5] P. Cheilaris, E. Specker and S. Zachos, Neochromatica, Comment. Math. Univ. Carolin. 51 (2010) 469-480.

[6] P. Cheilaris and G. Tóth, Graph unique-maximum and conflict-free colouring, J. Discrete Algorithms 9 (2011) 241-251. doi:10.1016/j.jda.2011.03.005

[7] J. Czap and S. Jendrol, Colouring vertices of plane graphs under restrictions given by faces, Discuss. Math. Graph Theory 29 (2009) 521-543. doi:10.7151/dmgt.1462

[8] G. Even, Z. Lotker, D. Ron and S. Smorodinsky, Conflict-free colorings of simple geometric regions with applications to frequency assignment in cellular networks, SIAM J. Comput. 33 (2003) 94-136. doi:10.1137/S0097539702431840

[9] I. Fabrici, S. Jendrol' and M. Vrbjarová, Unique-maximum edge-colouring of plane graphs with respect to faces, Discrete Appl. Math. 185 (2015) 239-243. doi:10.1016/j.dam.2014.12.002

[10] R. Glebov, T. Szabó and G. Tardos, Conflict-free coloring of graphs. arXiv: 1111.5501.

[11] M. Katchalski, W. McCuaig and S. Seager, Ordered colourings, Discrete Math. 142 (1995) 141-154. doi:10.1016/0012-365X(93)E0216-Q

[12] A.V. Kostochka, M. Kumbhat and T. Euczak, Conflict-free colorings of uniform hypergraphs with few edges, Combin. Probab. Comput. 21 (2012) 611-622. doi:10.1017/S0963548312000156

[13] A. Kündgen and R. Ramamurthi, Coloring face-hypergraphs of graphs on surfaces, J. Combin. Theory Ser. B 85 (2002) 307-337. doi:10.1006/jctb.2001.2107

[14] J. Pach and G. Tardos, Conflict-free colorings of graphs and hypergraphs, Combin. Probab. Comput. 18 (2009) 819-834. doi:10.1017/S0963548309990290

[15] R. Ramamurthi and D.B. West, Maximum face-constrained coloring of plane graphs, Discrete Math. 274 (2004) 233-240. doi:10.1016/j.disc.2003.09.001

[16] S. Smorodinsky, Conflict-free coloring and its applications. arXiv: 1005.3616 .

Received 23 September 2014

Revised 28 April 2015

Accepted 28 April 2015 